Rapid Thermal Analysis of Energetic Materials with Microfabricated Differential Scanning Calorimeters

C. M. Spadaccini, E. V. Mukerjee, J. Lee, W. P. King

March 18, 2009

Transducers 2009

Denver, CO, United States

June 21, 2009 through June 25, 2009 
This document was prepared as an account of work sponsored by an agency of the United States government. Neither the United States government nor Lawrence Livermore National Security, LLC, nor any of their employees makes any warranty, expressed or implied, or assumes any legal liability or responsibility for the accuracy, completeness, or usefulness of any information, apparatus, product, or process disclosed, or represents that its use would not infringe privately owned rights. Reference herein to any specific commercial product, process, or service by trade name, trademark, manufacturer, or otherwise does not necessarily constitute or imply its endorsement, recommendation, or favoring by the United States government or Lawrence Livermore National Security, LLC. The views and opinions of authors expressed herein do not necessarily state or reflect those of the United States government or Lawrence Livermore National Security, LLC, and shall not be used for advertising or product endorsement purposes. 


\title{
RAPID THERMAL ANALYSIS OF ENERGETIC MATERIALS WITH MICROFABRICATED DIFFERENTIAL SCANNING CALORIMETERS
}

\author{
Christopher M. Spadaccini ${ }^{1 *}$, Erik V. Mukerjee ${ }^{1}$, Jungchul Lee ${ }^{2}$, and William P. King ${ }^{2}$ \\ ${ }^{1}$ Center for Micro and Nano Technology, Lawrence Livermore National Laboratory, \\ Livermore, CA, USA \\ ${ }^{2}$ Department of Mechanical Science and Engineering, University of Illinois at Urbana-Champaign, Urbana, IL, \\ USA
}

\begin{abstract}
This paper introduces a class of single crystal silicon micro-scale differential scanning calorimeters for rapid detection and thermal characterization of energetic materials. The suspended membrane micro hotplates have fast time response and high sensitivity, which enables thermal measurements of melting endotherms and deflagration exotherms in energetic materials such as RDX and TNT. The potential exists for sensitivities in the picogram range with thermal scans in the 10's of milliseconds and controlled thermal cycling faster than $10^{6 \circ} \mathrm{C} / \mathrm{sec}$.
\end{abstract}

\section{KEYWORDS}

differential scanning calorimeter, energetic materials, micro hotplate

\section{INTRODUCTION}

Differential Scanning Calorimetry (DSC) is a thermoanalytical technique used to identify and characterize phase transitions and chemical reactions in various materials by directly measuring the heat evolved or absorbed in the process. DSC has been used to analyze energetic materials for many years [1]; however this tool is large (bench-scale), slow (thermal scans take hours), and requires large sample sizes $(\sim \mathrm{mg})$ making it inadequate for field detection applications. There are substantial benefits to miniaturizing a DSC-type system including improved sensitivity (small sample size, $\mu \mathrm{g}$-ng), ultra-fast measurements (10's of ms), low false positives, possibility of detecting unknown energetic materials, and portability.

A microfabricated version of a DSC allows for rapid trace detection and identification of known energetic materials as well as detection of unknown materials. Functionally, the $\mu \mathrm{DSC}$ is similar to its macroscale counterpart, detecting and differentiating oxidation exotherms and other thermal phenomena. This sensor concept takes advantage of the defining characteristic of energetic materials by measuring and quantifying their energy release. This would greatly reduce the probability of false positives, since common chemical interferents release significantly less energy upon thermal actuation. Miniaturizing and microfabricating the DSC could improve sensitivity and speed of analysis by orders of magnitude due to the greatly reduced thermal mass of the sensing element.

This paper reports the first rapid and direct thermal measurements of energetic materials in an all-silicon micro-scale DSC. Previous attempts at energetic materials characterization and detection in thermal microsystems involved deflection measurements of heated microcantilevers which cannot deconvolve force induced deflection from thermally induced signals; nor do they provide temperature scanning capability $[2,3]$.

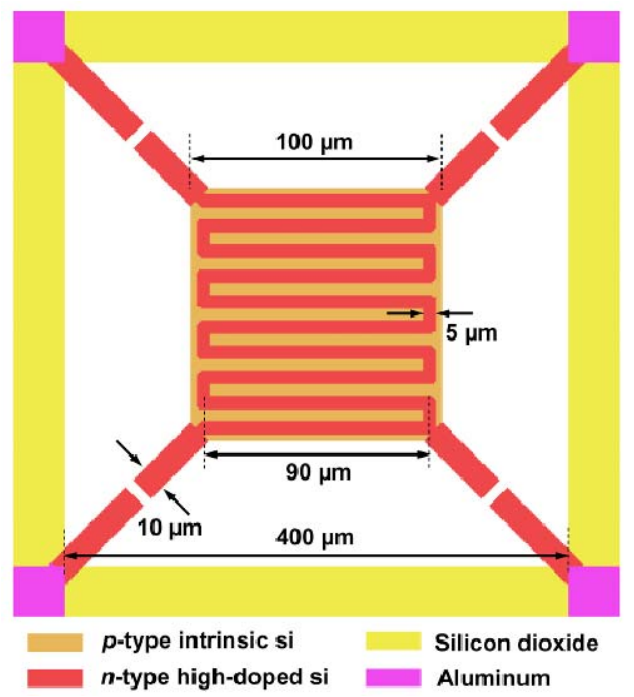

Figure 1. Design of the microfabricated calorimeter. A serpentine heater track runs from one tether to another through the island that will serve as a heater and sensor platform. Two tethers on the left are for heating and two tethers on the right for sensing.

\section{DESIGN AND FABRICATION}

Figure 1 shows the design of our microfabricated calorimeter. This design consists of a square silicon heater/thermometer element, suspended above a cavity by four electrically conductive tethers. A serpentine track of doped silicon runs across the membrane to provide heating. The cavity side length, heater side length, and tether width are $400 \mu \mathrm{m}, 100 \mu \mathrm{m}$, and $10 \mu \mathrm{m}$, respectively. A detailed heat transfer analysis for this device and its design rationale was presented by Lee et al. [4].

The electrical resistance of doped silicon resistors depends on the carrier mobility which is a strong function of doping concentration and temperature. Highly doped resistors exhibit low thermal noise and short heating time 
constants. Low doped resistors provide good temperature sensing characteristics. To choose a doping concentration for the silicon micro hotplate, the electrical resistance and thermal coefficient of electrical resistance were considered since they affect thermal noise, heating time constant, and sensitivity of the resistive thermometer. In addition, doped resisters have a nonlinear temperature dependence of electrical resistance, where the maximum electrical resistance is a function of the doping concentration. The temperature corresponding to the turn-over point increases as the doping concentration increases. To guarantee low thermal noise, rapid heating, and high-temperature sensitivity, a doping concentration between $10^{20}$ and $10^{21} / \mathrm{cm}^{3}$ is recommended since the thermal coefficient of electrical resistance of the doped silicon resistor is comparable to that of metal contacts [4].

The fabrication process started with a $p$-type silicon-oninsulator wafer of orientation $<100>$ with a silicon device layer of $340 \mathrm{~nm}$. The tethered membrane structures were defined using an inductively coupled plasma (ICP) etcher. The serpentine heater and tethers were patterned with photoresist and implanted with $2.51 \times 10^{16} \mathrm{~cm}^{-2}$ of phosphorous at $180 \mathrm{keV}$. Electron beam evaporation in conjunction with a lift-off process defined the aluminumdoped silicon contacts. The backside of the wafer was patterned with a thick photoresist and etched using ICP.

Finally, $400 \mathrm{~nm}$ of PECVD nitride was deposited on the front side for passivation. The major fabrication steps are shown in figure 2 .

Figures 3(a) and (b) show scanning electron micrographs of the fabricated micro hotplates having a heater side length of $100 \mu \mathrm{m}$. In a $\mu \mathrm{DSC}$ unit cell, there are four identical micro hotplates so that any two can perform differential measurements. Each unit cell was wire-bonded to a 28 pin dual-in-line package (figure 3(c)).

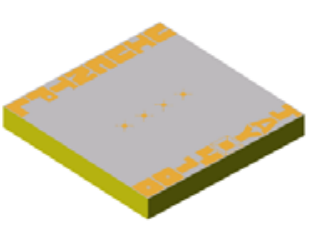

(a) Etch device layer

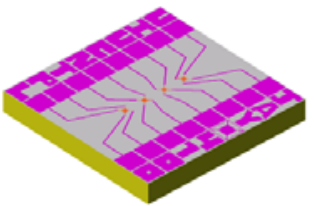

(c) Metallization



(f) Passivation

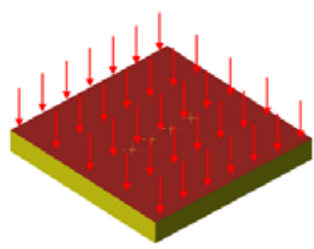

(b) Implantation



(d) ICP etch

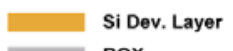

BoX

Si Handle

PR



$\mathrm{Si}_{3} \mathrm{~N}_{4}$

Doped Si

$\downarrow \downarrow \downarrow \downarrow$ Implantation
Figure 2. Five major fabrication steps
The entire assembly was placed into a custom built chamber with electrical and gas I/O capabilities for inert gas/vacuum environment electrical testing. Figure 3(d) shows the packaged unit cell and test chamber.

DC electrical characterization was performed. The fabricated microhotplates have a doped silicon resistor which can function as either a heater or a resistive thermometer. First, the resistor was operated as a heater connected to an $8 \mathrm{k} \Omega$ sense resistor. Figure 4 shows the electrical resistance as a function of heater temperature. The heater temperature was measured at the center with laser Raman thermometry while the micro hotplate was resistively-heated [5]. The temperature coefficient of resistance was $1.223 \times 10^{-3} \mathrm{~K}^{-1}$. After this characterization, the temperature of the calibrated microcalorimeter can be monitored based on the heater resistance $[4,5]$.

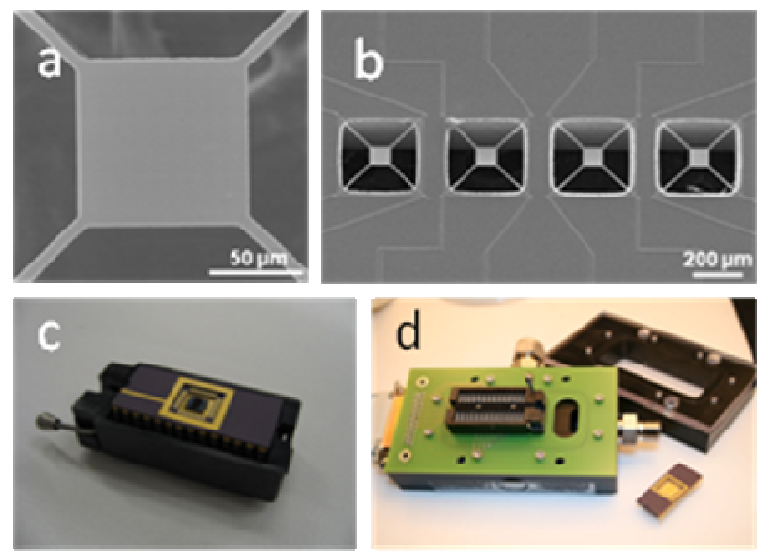

Figure 3. Scanning electron micrographs of the fabricated microcalorimeter. (a) A close up of one device. (b) A $\mu D S C$ unit die having four identical micro hotplates. (c) A picture showing a $\mu \mathrm{DSC}$ unit in a dual-inline package. (d) Environmental test chamber.

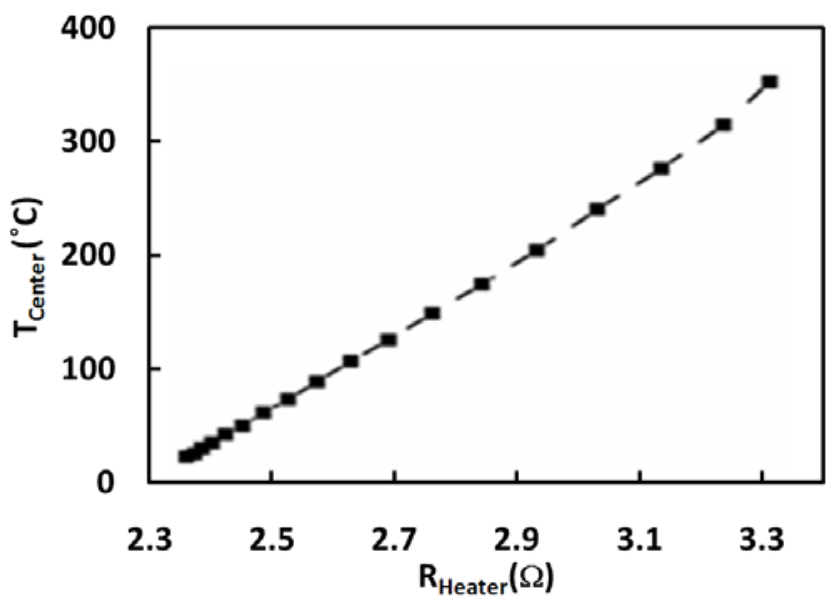

Figure 4. Temperature calibration. 


\section{EXPERIMENTAL SETUP}

After packaging and characterization, the $\mu \mathrm{DSC}$ devices were tested with energetic materials. The experimental setup for these tests included a arbitrary waveform generator for providing the input voltage ramp to the devices, an oscilloscope, a high speed data acquisition system, and a laptop computer for control and data collection. A typical input voltage ramp for testing of energetic materials was $50-100 \mathrm{~V} / \mathrm{sec}$, corresponding to a $3000-6000^{\circ} \mathrm{C} / \mathrm{sec}$ ramp.

Sample loading was accomplished by first evaporating a droplet of acetonitrile solvent which contained dissolved explosive material. A film of either RDX or TNT remained and was then removed by a silicon microneedle device affixed to a wire bonding stage. Using the stereoscope and manual controls of the wire bonder, the sample was maneuvered into position over the microcalorimeter and deposited.

\section{ENERGETIC MATERIALS TESTING}

The microcalorimeters were operated without a sample present both before and after all tests with energetic material. This established a baseline for comparison indicated by the triangular symbols in figures 5,6 , and 7 . Typically for these reference runs, the heater resistance ramped from $\sim 4000 \Omega$ to $\sim 5500 \Omega$, corresponding to $\sim 25$ $450^{\circ} \mathrm{C}$. Around $5500 \Omega$ the resistance changes slope due to intrinsic carrier based "thermal runaway".

The solid trace shown in figures 5,6 , and 7 , is the sensor response to an RDX sample during thermal scanning. In this case, the sensor was uncalibrated and resistance versus time is shown. A linear voltage ramp of $80 \mathrm{~V} / \mathrm{sec}(0-8 \mathrm{~V}$ in $100 \mathrm{~ms})$ was applied and generated a temperature ramp of $\sim 4000^{\circ} \mathrm{C} / \mathrm{sec}$. Experiments with TNT were also conducted yielding similar results.

The endothermic melting transition is observed at $\sim 4400 \Omega$ where the RDX curve deviates from the reference trace. During the melting phenomenon, the resistance (and temperature) remains roughly constant as would be expected during a phase change. By melting the RDX, the thermal contact between sensing element and sample improves significantly. As the sample continues to heat, the resistance (and temperature) rises on a parallel yet lower curve than the reference. This is due to the additional thermal mass of the sample. The exothermic deflagration of the RDX (relative to the reference data) can be seen in figures 5 and 7. After passing through the maximum resistance, a sharp yet brief decrease in the resistance is observed which corresponds to the RDX deflagration. Although capturing the deflagration signature on the downward sloping side of the resistance curve is not ideal, it qualitatively indicates that the deflagration exotherm is measurable in a silicon microsystem of this type.

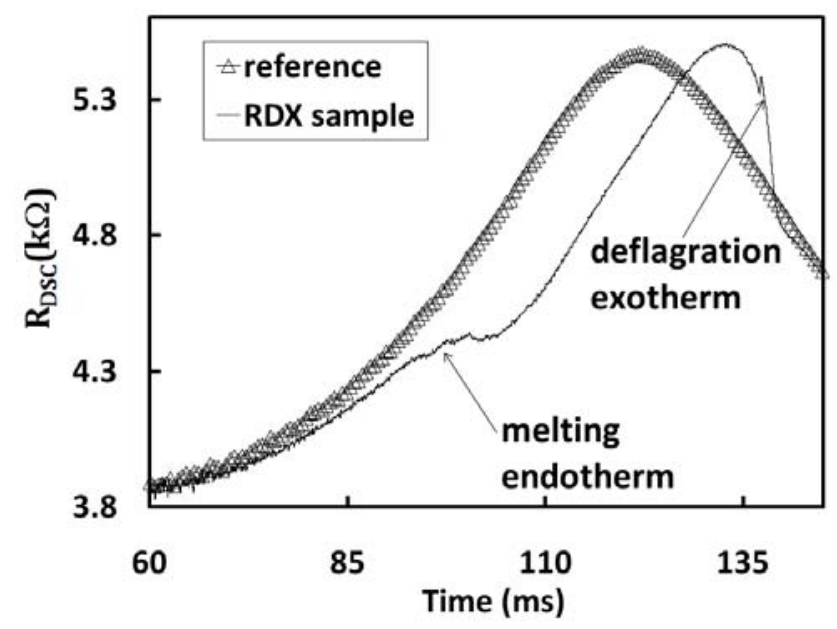

Figure 5. Calorimeter resistance vs. time for reference and $R D X$ sample showing melting endotherm and deflagration exotherm.



Figure 6. RDX melting endotherm and reference calorimetric trace.



Figure 7. RDX deflagration exotherm and reference calorimetric trace. 

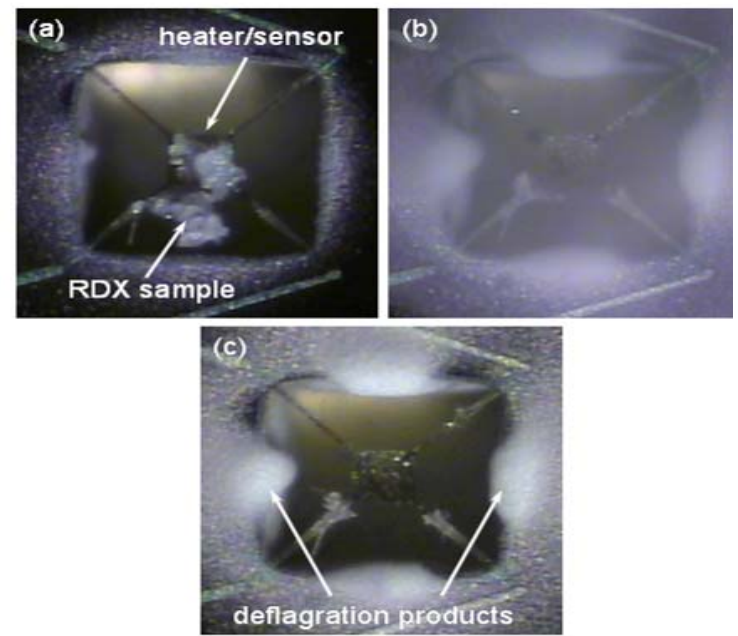

Figure 8. (a) Before, (b) during, and (c) after RDX deflagration on microcalorimeter.

Figure 8 shows photographs of the $\mu \mathrm{DSC}$ with RDX. Figure 8(a) is prior to operation and a "flake" of white RDX can be seen resting on the square sensing element. Based on size, we calculate that the RDX fragments used in these tests are approximately 50-100 ng. Figure 8(b) shows a cloud of RDX reaction product which is generated during deflagration and Figure 8(c) is post operation. RDX debris (products of the reaction) is deposited around the sensor perimeter. Typically, very little debris is left on the sensing element itself and can be thermally desorbed.

\section{DISCUSSION}

We can estimate the theoretical detection limit (in grams of energetic material) of a micro-scale DSC device via the simple relation

$$
\mathrm{HE}(\mathrm{g})=\frac{m_{S i} \cdot C_{p(S i)} \cdot T_{R E S}}{H_{c(H E)}}
$$

where $\mathrm{HE}(\mathrm{g})$ is the minimum detectable mass, $m_{S i}$ is the mass of silicon used in the sensor, $C_{p(S i)}$ is the heat capacity of silicon, $T_{R E S}$ is the thermal resolution of the sensor, and $H_{c(H E)}$ is the heat of combustion of the energetic sample. The assumptions used include:

1. No convective/conductive/radiative thermal losses in the system. All heat evolved from deflagration is coupled directly into the sensor element.

2. The sensor geometry is square and $\sim 200 \mathrm{~nm}$ thick.

3 . The sensing element is made primarily of silicon.

Based on this analysis, figure 9 shows sensitivity versus sensor size for a variety of energetic materials and a thermal resolution of $0.1^{\circ} \mathrm{C}$. From this plot it is clear that a micro-scale DSC can have theoretical sensitivities in the sub picogram range.

\section{SUMMARY AND CONCLUSIONS}

Micro-scale DSC devices offer the capability to detect and characterize trace quantities of energetic materials

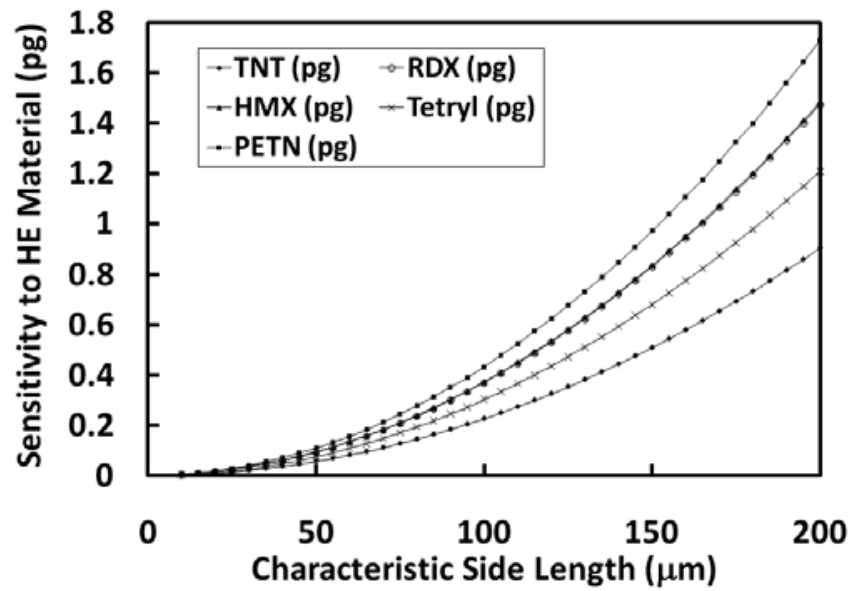

Figure 9. Theoretical sensitivity of a $\mu \mathrm{DSC}$ device.

rapidly and with low false positives. This work has shown this capability by fabricating micro hotplate calorimetric devices and testing them with both RDX and TNT. This proof of concept indicates that there exists great potential to improve performance.

\section{ACKNOWLEDGEMENT}

The authors gratefully acknowledge the financial support of the DARPA Microsystems Technology Office, Program Manager Dr. T. Kenny.

Portions of this work were performed under the auspices of the U.S. Department of Energy by Lawrence Livermore National Laboratory under Contract DE-AC5207NA27344. LLNL-PROC-XXXXXX

\section{REFERNCES}

[1] "A study on thermal decomposistion behaviors of PETN, RDX, HNS and HMX," J-S. Lee, et al., Thermochimica Acta, 392-393 (2002), pp. 173-176.

[2] "A microsensor for trinitrotoluene vapour," L.A. Pinnaduwage, et al., Nature, 425 (2003), pg. 474.

[3] "Detection of trinitrotoluene via deflagration on a microcantilever," L.A. Pinnaduwage, et al., J. Applied Physics, 95 (2004), pp. 5871-5875.

[4] "Differential Scanning Calorimeter Based on Suspended Membrane Single Crystal Silicon Microhotplate," J. Lee, C. Spadaccini, E. Mukerjee, W. King, J. Microelectromech. Syst., 17 (2008), pp.15131525.

[5] "Electrical, thermal and mechanical characterization of silicon microcantilever heaters," J. Lee, T. Beechem, T.L. Wright, et al., J. Microelectromech. Syst., 15 (2006), pp. 1644-1655.

\section{CONTACT}

* Christopher M. Spadaccini, tel: +1-925-423-3185; spadaccini2@1lnl.gov 